\title{
Pediatric scoliosis predictive blood tests: progress and challenges for clinicians
}

\author{
Alain Moreau*, Marie-Yvonne Akoume, Anita Franco, Isabelle Turgeon, Maryam Taheri, Ginette Lacroix, \\ Ginette Larouche, Benoît St-Jacques, Da Shan Wang, Hubert Labelle, Benoît Poitras, Charles-Hilaire Rivard, \\ Guy Grimard, Stefan Parent, Jean A Ouellet
}

From 7th International Conference on Conservative Management of Spinal Deformities Montreal, Canada. 20-22 May 2010

\section{Purpose}

There are great needs for innovative pharmacotherapies in combination with clinical tests to identify asymptomatic children at risk of developing scoliosis and symptomatic ones to predict who may be at risk of scoliotic curve progression. Early detection of scoliosis is critical to broaden the range of treatment options and increases effectiveness. Currently, there are no FDA cleared "presymptomatic" diagnostic tests available for assessing scoliosis in paediatric patients. We have developed a cell-based screening assay for the early diagnosing of presymptomatic subjects and a biochemical blood test for asymptomatic individuals and patients at different disease stage.

\section{Methods}

Peripheral blood samples for AIS patients, asymptomatic children and control subjects were collected in blood collection tubes containing EDTA and then centrifuged on a Ficoll-Plaque solution to obtain peripheral blood mononuclear cells (PBMCs) and plasma. Gi-coupled receptor signal transduction was measured by cellular dielectric spectroscopy (CDS) in presence of varying concentration of melatonin or other ligands. Plasma concentrations of OPN and sCD44 were measured by ELISA methods adapted to be performed on a robotic platform.

\section{Results}

In a cross-sectional clinical study, we have shown that mean plasma OPN levels were significantly increased in AIS patients $(n=320)$ and correlated with disease severity with average values of $743 \pm 326$ and $975 \pm 389 \mathrm{ng} / \mathrm{ml}$ for moderate and severe spinal deformities, respectively, when compared to the healthy control group (568 \pm $216 \mathrm{ng} / \mathrm{ml} ; \mathrm{n}=120$ ). Elevated plasma OPN levels were also found in the asymptomatic at-risk group (871 \pm $387 \mathrm{ng} / \mathrm{ml} ; \mathrm{n}=87$ ), suggesting that these changes precede scoliosis onset. Data obtained using PBMCs revealed a melatonin signaling impairment only in IS patients at different disease stages when compared to healthy controls showing a high specificity $(100 \%)$ and sensitivity $(100 \%)$. Risk of developing a scoliosis in asymptomatic children was determined by CDS in 33\% of asymptomatic children at risk.

\section{Conclusion}

Both tests have a unique advantage since they can be performed without any prior knowledge of mutations in any defective genes causing AIS. The standard of care for scoliosis has not changed in any significant manner in decades. Patients today are treated in a substantially similar manner to those twenty or thirty years ago observation, bracing, and fusion as a last resort. Our diagnostic blood-assay may have the potential to change the way scoliosis patients are diagnosed and treated.

Published: 10 September 2010

doi:10.1186/1748-7161-5-S1-03

Cite this article as: Moreau et al:: Pediatric scoliosis predictive blood tests: progress and challenges for clinicians. Scoliosis 2010 5(Suppl 1):O3.

Sainte-Justine Hospital, Montreal, Canada

Full list of author information is available at the end of the article 